\title{
La pantalla que nos \\ separa: \\ docentes y \\ estudiantes \\ en tiempos \\ de pandemia, \\ conectados/as y \\ desconectados/as
}

Justo Díaz-Ortiz y Yessenia Fabiola López-de Jesús²

1 Profesor de Asignatura de la Licenciatura en Ciencias de la Educación, adscrito al Instituto de Ciencias de la Educación de la Universidad Autónoma "Benito Juárez" de Oaxaca. Av. Universidad s/n, col. Cinco Señores, Oaxaca, Oax.

2 Profesora de Asignatura de la Licenciatura en Ciencias de la Educación, adscrita al Instituto de Ciencias de la Educación de la Universidad Autónoma "Benito Juárez" de Oaxaca. Av. Universidad s/n, col. Cinco Señores, Oaxaca, Oax. 


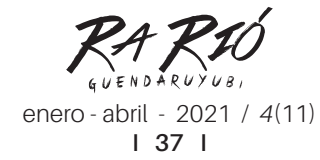

| 37 |

\section{Resumen}

El presente artículo reúne opiniones y reflexiones en torno a la complejidad de los procesos educativos en el contexto de la contingencia sanitaria del Covid-19 por la que atravesamos y cómo a partir de su declaratoria como pandemia reveló grandes carencias en el ámbito educativo de nuestro país, tal es el caso de la puesta en marcha de nuevas formas 0 modalidades para seguir con el desarrollo de las actividades escolares, en todos los tipos y niveles educativos. De este modo, analizamos claramente las dificultades de las condiciones bajo las cuales maestros/as y estudiantes lidian con el desarrollo de sus tareas educativas frente a un monitor o pantalla, lo cual permite, por un lado, estar tan cerca, conectados/as a una red que nos permite oírnos, vernos y mirarnos, y por otro, estar tan alejados/as de una dinámica que demanda relacionarnos cara a cara, hablamos de la presencialidad necesaria en el proceso educativo.

Palabras clave: Práctica docente, proceso educativo, conexión y desconexión, contingencia, pandemia, docencia.

\section{Abstract}

This paper integrates opinions and reflections on the complexity of the educational processes in the context of the Covid-19 sanitary contingency. It examines how, since it was declared a pandemic, Covid-19 has revealed great deficiencies in the Mexican educational field, as is the case of the newly implemented ways that are meant to continue with the development of school activities, in all educational levels. This is how we observe, clearly, how difficult the conditions are for teachersandstudents to conclude their educational tasks in front of a computer screen, which on one hand allows them to feel close among themselves, connected to a network that lets them hear and see each other, and on the other hand, by being so far away from a process that demands a faceto-face relationship, it allows us to study how integral the face-toface dynamic is to the teaching process.

Keywords: Teaching practice, educational process, connection and disconnection, contingency, pandemic, teaching

La pantalla que nos separa: docentes y estudiantes en tiempos de pandemia conectados/as y desconectados/as Diaz-Ortiz \& López-de Jesús 

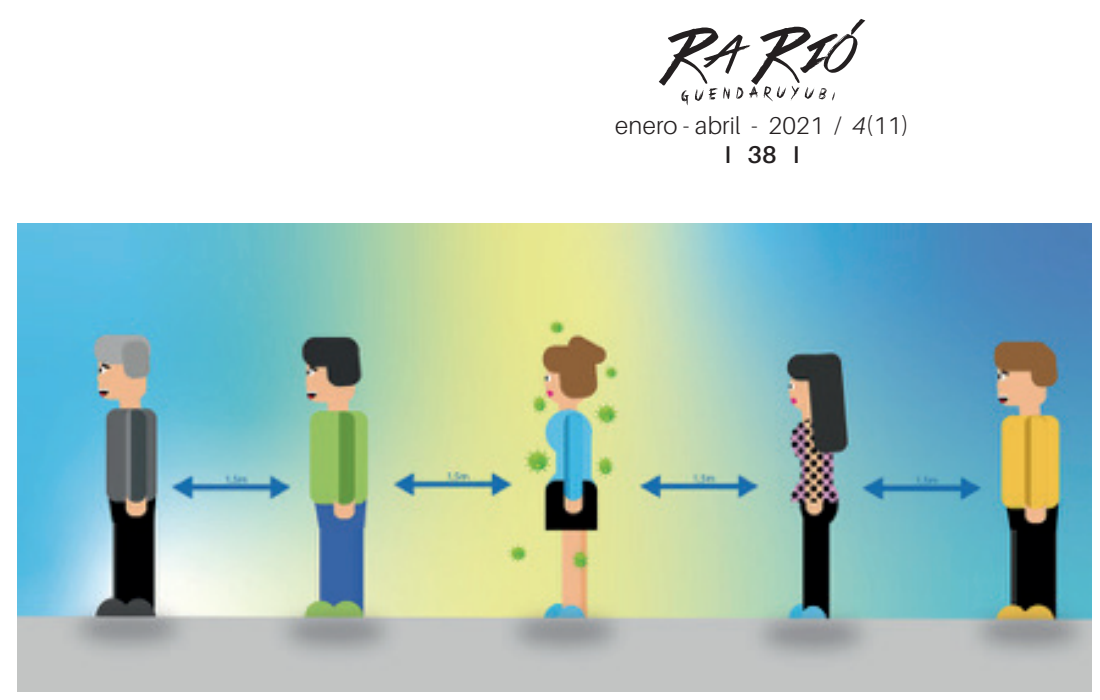

Distanciamiento social Fuente: Pixabay, 2021.

\section{El nuevo orden social}

A raíz de los acontecimientos vividos en los últimos años y específicamente a partir de la aparición y propagación del COVID-19 a escala mundial, una afirmación que puede hacerse con toda seguridad es que las cosas cambian, las miradas son diferentes, y con ello se afectan las actividades realizadas en los múltiples ámbitos de nuestra vida: económico, cultural, político y, desde luego, el educativo, al que se le apuesta para mejorar la conciencia colectiva. Al respecto, sugerimos ver el video "La Tierra nos está hablando" ${ }^{3}$ en el cual se plantea un conjunto de situaciones importantes que invitan a la reflexión en todas las esferas de la vida social, que hacen pensar profundamente en las múltiples formas de estar y ser en el entorno que nos rodea.

Complementando lo anterior, nos parece relevante mencionar diversos desafíos que López (2020) planteó en su conferencia titulada

\footnotetext{
${ }^{3}$ El recurso se encuentra alojado en el siguiente enlace: https://www.youtube.com/watch?v=kWTHU8kXF-c 

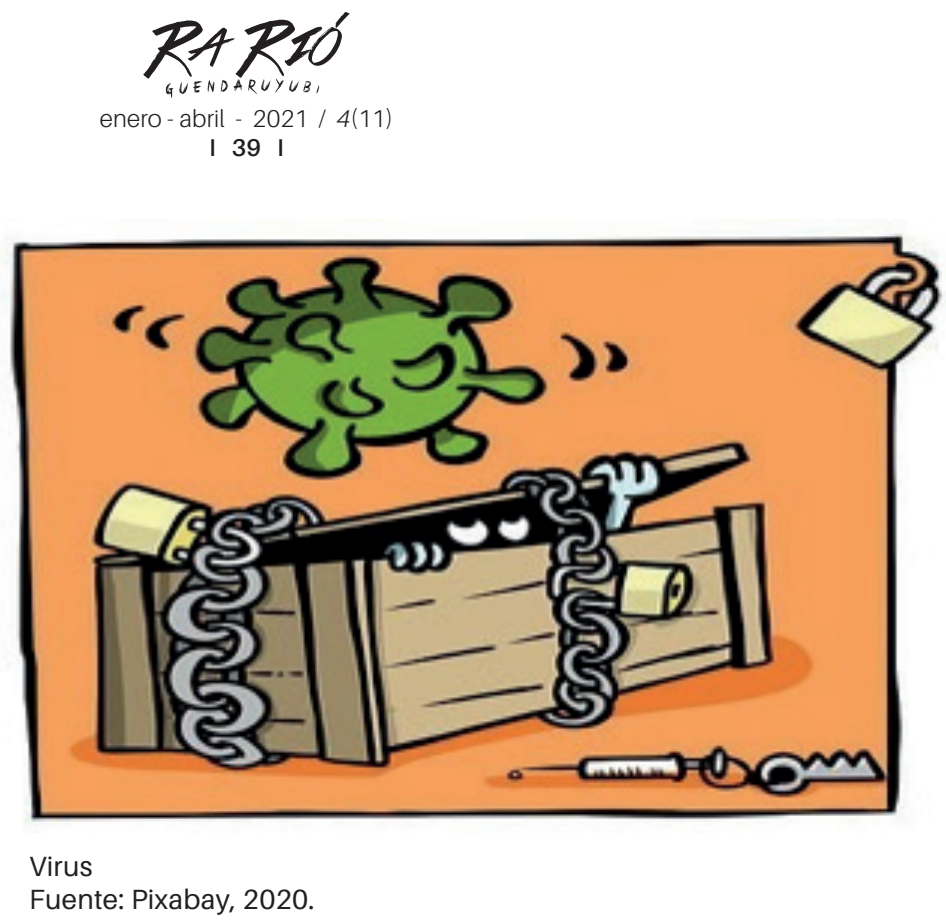

"Re-danzar el mundo. Panorama global del maestro en el 2020", en relación con las nuevas dinámicas en todos los campos de acción humana. Así, se habla de retos en el aspecto ecológico, desde el cual se reflexiona en torno al hecho de que un diminuto organismo exhibió y sigue exhibiendo la vulnerabilidad de la especie humana frente a la devastadora naturaleza y, por ende, a meditar cuidadosamente en su preservación y en la necesidad de unir esfuerzos para la sobrevivencia colectiva. Del mismo modo, se establece un nuevo desafío antropológico, motivado por la pregunta ¿qué es el ser humano hoy?, reconociendo que -como se expresa en algunas ocasiones- las tragedias "sacan lo mejor o lo peor de las personas"; es decir, a partir de la vivencia de situaciones complicadas, los seres humanos proyectamos los valores o antivalores que nos dan sentido y que guían nuestras actuaciones. Aunado a la idea anterior, el autor antes citado reflexiona sobre desafíos éticos, orientados a los valores en crisis y las interrogantes respecto del sentido de la vida, incluso sobre la cuestión de nuevas formas de convivencia cuando parece que el optimismo y la esperanza se ven como puntos lejanos en el horizonte, lo que se liga estrechamente con los cuestionamientos: ¿Qué es el ser humano actualmente?, ¿qué valores y principios son necesarios hoy y cómo enseñarlos?, ¿se requiere reformular y aplicar una nueva ética que permita vivir de un modo más pleno, empático y consciente?

Por su parte, el desafío en el que podemos cavilar primeramente es ¿qué se puede considerar como "verdad" actualmente?, en un contexto en el cual el conocimiento científico se desplaza y se esparce información sobre diversos temas por medio de distintas redes sociales, proveniente de muchos sitios y que se asume como cierta a partir del nivel de 

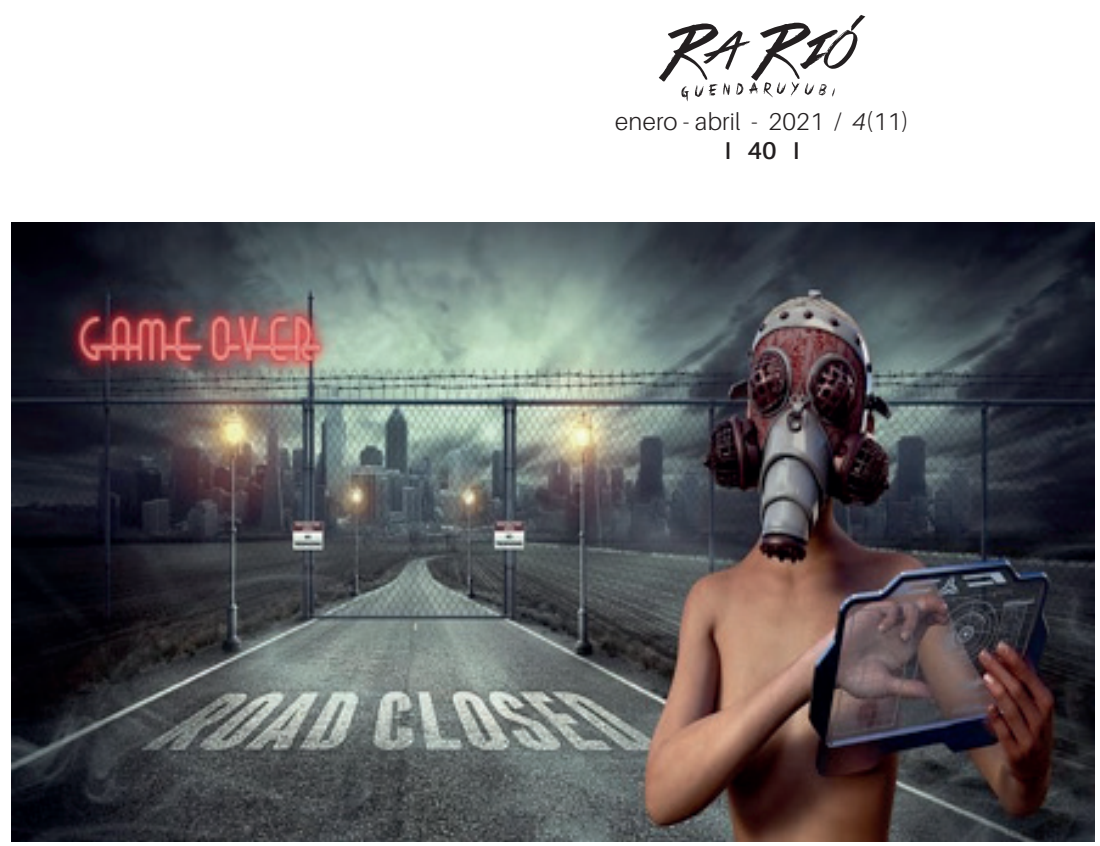

Pandemia-epidemia coronavirus

Fuente: Pixabay, 2020.

popularidad que alcance. Una noticia se mide como verdadera por el número de veces que ha sido retuiteada o por el número de likes y "me encanta" que recibe. Así, vivimos simultáneamente una segunda pandemia, la de las fake news.

Por otro lado, se plantea desde una perspectiva social afrontar la crisis económica del sistema-mundo, lo cual demanda repensar en otras propuestas teóricas desde las cuales se maneja la economía en el planeta, otras formas de hacer negocio, generar ingresos, comercializar productos, etcétera.

En otro orden de ideas, López también expresó en su conferencia que se nos coloca ante un reto de corte tecnológico, que se desarrolla a partir de una pregunta central: ¿Estamos ante un inminente fin de la educación presencial? En palabras del autor, la respuesta es un rotundo no; sin embargo, invita a entender claramente el sentido que tienen tanto la educación presencial como la virtual, además de reflexionar profundamente sobre la relación entre tecnología, aprendizaje y desarrollo humano, buscando en todo momento el equilibrio de estos elementos en la concreción de los procesos didácticos.

En lo que compete a la gestión, el conferencista expresa la necesidad de repensar cómo se mueven los sistemas educativos, los cuales en estos tiempos han caído en una crisis aguda en cuanto a los procesos y los resultados. Ante ello, es importante reconstruir un nuevo pacto y alianza entre la escuela, la familia y la sociedad, así como en las nuevas maneras de educar y enseñar que permitan la estrecha vinculación de todos/as los/as participantes en la formación académica. 


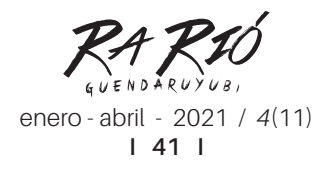

Sumado a lo anterior, continuamente se ha mencionado que un propósito primordial y vigente en la educación es innovar los procesos de enseñanza y aprendizaje, renovar y reinventar permanentemente las prácticas, acciones, hábitos y aprendizajes que se promueven a lo largo de la vida de las personas. Esta aspiración se ha mantenido en la agenda de pendientes a considerar por las autoridades educativas, dado que aún resultan necesarios apoyos significativos al sector educativo que permitan enfrentar contingencias como la que vivimos actualmente de forma más preparada y con mejores herramientas, afectando lo menos posible la formación de millones de estudiantes; no obstante, en nuestro país existe un número considerable de escuelas sin áreas de computación, sin infraestructura, equipamiento, ni personal asignado para realizar dinámicas educativas en modalidades no convencionales. La educación a distancia es aún una ilusión en México.

Tal parece que la situación ante la que nos colocó la pandemia a partir de marzo de 2020, como bien se ha dicho en diferentes foros, conversatorios, paneles y eventos académicos realizados desde entonces, agudizó aún más la fragilidad del sistema educativo mexicano. No es que con la declaratoria de pandemia por parte de las autoridades sanitarias a nivel nacional las estructuras educativas se hayan fracturado; desde tiempo atrás, la educación en nuestro país se encuentra en un estado doliente, la crisis es su condición natural y normal. La pandemia generada por el COVID-19 sólo vino a canalizar la educación directamente al área de cuidados intensivos de las clínicas sociales.

En este escenario, la educación brindada en todos los tipos y niveles experimentó un notable y nuevo cambio de rumbo, pues para evitar la propagación de los contagios se decidió suspender las clases presenciales, tomando diversas alternativas para impartirlas en otras modalidades: a distancia, virtual, en línea o por medio de los programas televisivos "Aprende en casa", esta última estrategia fue definida por las autoridades educativas nacionales e implementada para iniciar el ciclo escolar 2020-2021, principalmente en la educación básica. 


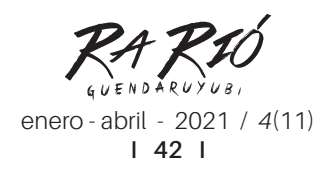

\section{Los procesos educativos en tiempos de pandemia}

Las Tecnologías de la Información y la Comunicación anteriormente se definían como un componente importante en el campo educativo, pues brindaban herramientas electrónicas a los/as actores/actrices educativos/as para mejorar o acompañar los procesos de enseñanza y aprendizaje. A la fecha, para continuar con el desarrollo de las clases en todos los tipos, niveles y modalidades se han aprovechado estos recursos y aplicaciones digitales, a través de los cuales se ha mantenido contacto con las y los estudiantes, colocando a este sector, así como al profesorado, frente a importantes retos y nuevas dinámicas que han evidenciado áreas de oportunidad y un campo para cultivar otros aprendizajes desde casa y maneras de hacer docencia.

A propósito de este punto, se retoma de la conferencia mencionada anteriormente, impartida por el Dr. Juan Martín López Calva, el hecho de que la contingencia sanitaria provocó que no sólo los/as maestros/as, sino que los distintos grupos de la población, tuvieran oportunidades para reconocer y desarrollar capacidades que seguramente estaban adormiladas o poco potencializadas, pero estando en cuarentena hubo la ocasión de ponerlas en práctica, en medio del aburrimiento y el ocio, precisamente para hacer más llevadero el encierro.

En este sentido, trasladar el aula a la casa nos impone serios cambios que no únicamente repercuten en el/la estudiante y el/la docente, sino que trastocan las actividades familiares de cada persona, desde acondicionar el espacio, programar los horarios de clases o propiciar condiciones que permitan el acceso adecuado a cada sesión de aprendizaje, debido a que en la mayoría de los hogares hay más de un/a estudiante que necesita conectarse a las clases en línea y en el caso de los/as profesores/as, deben impartir sus videoconferencias, programar diferentes tareas en las plataformas electrónicas, recibir trabajos vía correo electrónico, calificarlos, entre otras labores.

Las limitaciones económicas y digitales ante esta nueva situación se revelan de manera adversa en las familias mexicanas, pues se 


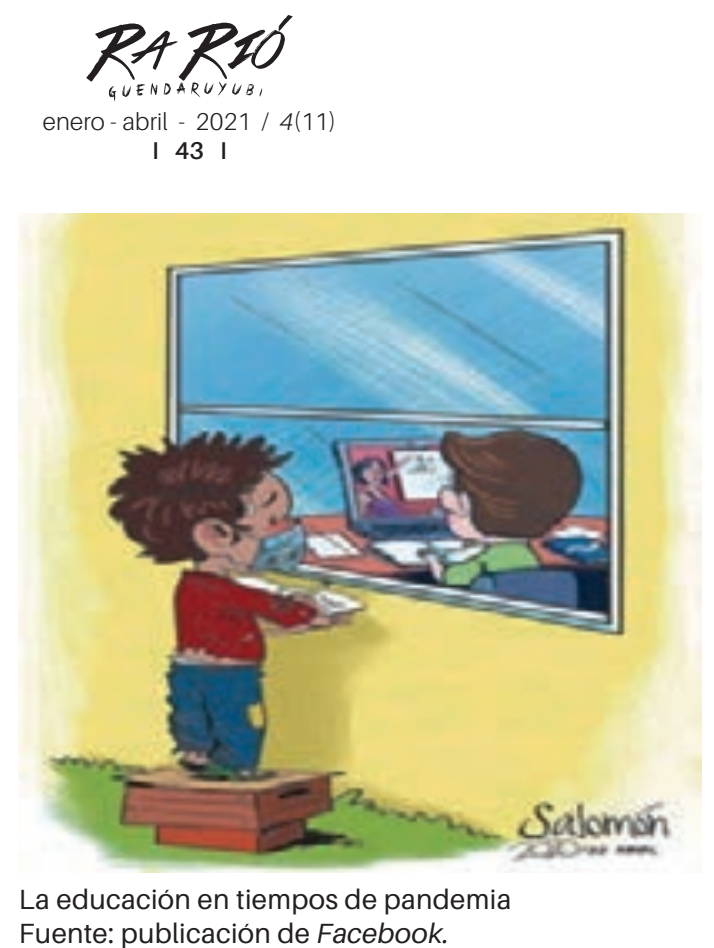

registran 80.6 millones de usuarios/as de internet, que representan $70.1 \%$ de la población de seis o más años, mientras que $76.6 \%$ de los/as habitantes del contexto urbano son consumidores/as de internet. En la zona rural, la población que utiliza la red se ubica en $47.7 \%$ a nivel nacional, según la Encuesta Nacional sobre Disponibilidad y Uso de Tecnologías de la Información en los Hogares (INEGI, 2019). En particular, la entidad oaxaqueña se integra por regiones rurales y urbanas.

\section{Entre la conexión y la desconexión}

No dejar a nadie atrás significa no dejar a nadie desconectado. António Guterres, Secretario General de las Naciones Unidas

La conectividad es un requisito primordial en este contexto, la ruta a la cual se voltea a ver para no romper con un ritmo de trabajo, porque la escuela, la enseñanza y el aprendizaje deben continuar, a fin de evitar mayor rezago educativo y profundizar aún más los baches que se han formado desde años atrás. Recuperando elementos vertidos por Puiggrós, Arroyo, Aguilar \& López (2020), en el foro virtual "Los sistemas educativos nacionales antesituaciones deemergencia", noseinterrumpióla conexión entre el sistema educativo y el sector de estudiantes, debido a que hubo una reacción rápida y eficiente, aunque no fue (y no es) generalizada, puesto que en muchas regiones no se cuenta con las condiciones y los recursos mínimos e indispensables para participar en procesos educativos bajo modalidades distintas a la escolarizada y presencial. Tal es el caso de nuestro estado, ya que la geografía determina la distancia 

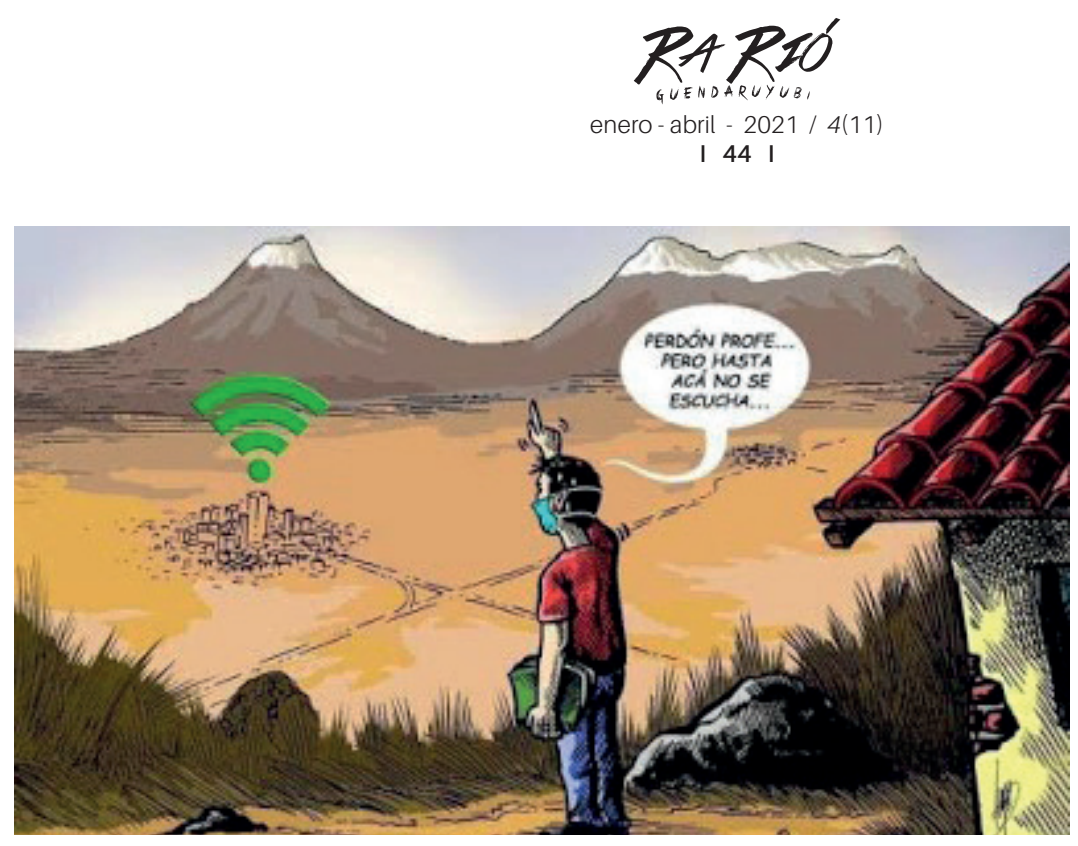

La desigualdad digital

Fuente: https://otra-educacion.blogspot.com/, 2020.

hacia escuelas que se encuentran muy alejadas del centro, además de la mala conectividad en las comunidades.

Así, la nueva dinámica escolar obligó a explotar y familiarizarse aún más con diferentes herramientas y recursos electrónicos, así como con aplicaciones y plataformas digitales, servicios de videotelefonía como Zoom, Meet, Teams, entre otros, con los cuales conectarnos y acercarnos para seguir educando y construyendo en el distanciamiento social. Es importante reconocer el valor y utilidad de todos los medios que tenemos a la mano para mantenernos en contacto; estamos lejos (físicamente), pero cerca (virtualmente) para avanzar en la adquisición de contenidos, desarrollo de habilidades y puesta en marcha de actitudes y valores.

Estamos, pues, entre la conexión, porque actualmente "todo el mundo" tiene alguna posibilidad para asistir y participar en la era virtual, teniendo a disposición un dispositivo (móvil, tableta, laptop) e internet (datos móviles, wifi, señal) y eso nos acerca, nos sentimos acompañados/as. Por otro lado, estamos ante la desconexión, porque docentes y estudiantes no sabemos qué ocurre detrás de la pantalla, literalmente, más aún cuando toda posibilidad de contacto e interacción se desvanece y diluye en la virtualidad (desde ver los rostros de los/as otros/as, escuchar las voces, observar los gestos, mirar comportamientos, percibir emociones, en suma, dar muestras e indicios de humanidad, y ni hablar si las cámaras y micrófonos están apagados), se hace de la clase un encuentro vacío, falto de significado y sentido. Los/as maestros/as nos quedamos hablando solos/as, tenemos la sensación de que nuestra enseñanza está dirigida hacia una pantalla y no a los/as estudiantes. Esta situación se torna compleja también, porque no es posible que alumnos y alumnas mantengan 

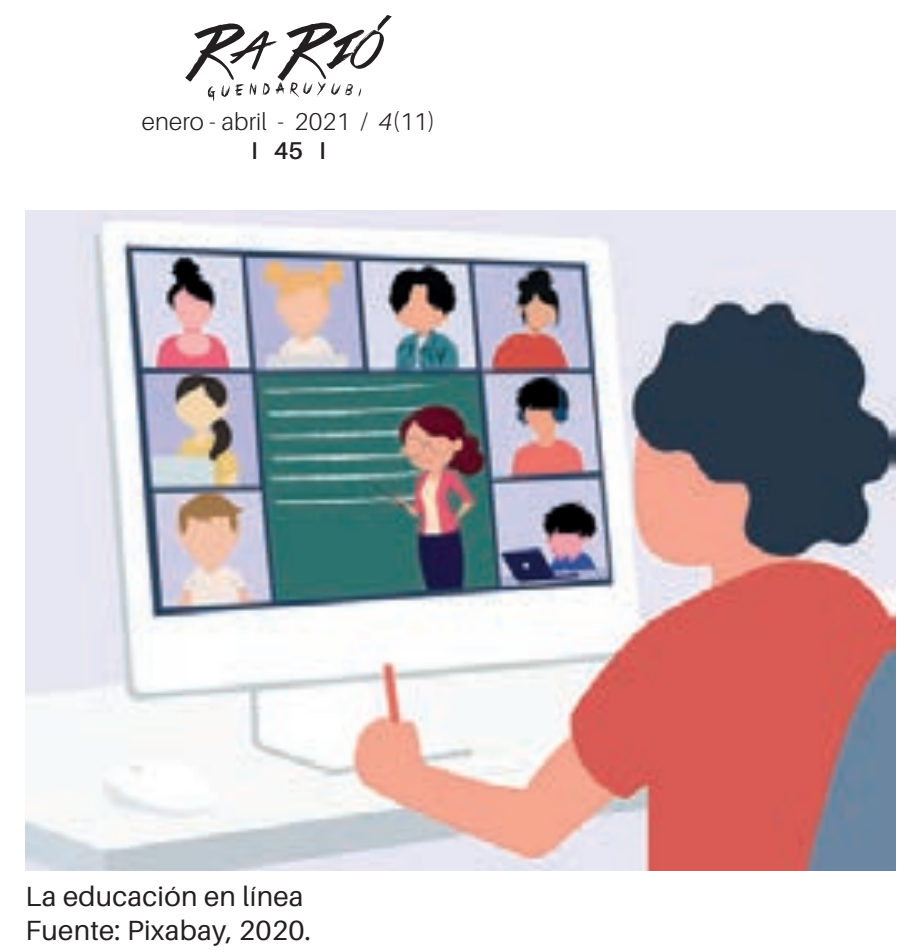

encendidas las cámaras todo el tiempo y se ven en la necesidad de apagarlas debido a las problemáticas que se presentan en cuanto a la saturación de la red al momento de hacer uso de la cámara y sonido de muchos dispositivos, pues la calidad de interacción disminuye, se distorsiona, se desconecta, "se va", no nos permite mirarnos.

Por ello, Hernández (2020), en la videoconferencia "El campo educativo en el contexto actual", se pronuncia en defensa de la escuela presencial, recuperando de este modo una de las aportaciones del padre de la didáctica, Juan Amós Comenio, resaltando también que la esencia del proceso educativo es "construirnos en la alteridad y en el contacto", así como la necesidad de que docentes y estudiantes nos veamos a la cara, establezcamos contacto visual, saber que nos estamos escuchando, tener la posibilidad de preguntar si los temas vistos han sido del agrado o no, significativos o no, detectar cómo se sienten los/as otros/as.

Actualmente, en medio de la pandemia, como docentes y estudiantes nos perdemos de esas interacciones que les imprimen sentido y significado a los procesos de enseñar y aprender. De este modo, se puede decir que la presencialidad no se sustituye con nada, porque ese factor permite la vinculación y acompañamiento entre los seres humanos, componentes necesarios en la tarea educativa.

Lo anterior nos lleva a recuperar una frase de Arthur C. Clarke que invita a la reflexión sobre el papel primordial que desempeñan maestras y maestros en el desarrollo de las acciones educativas: "Un profesor que puede ser sustituido por una máquina, debe ser sustituido" (TED Ideas worth spreading, 2007). Es decir, quien renuncia a refrendar su compromiso con la vocación docente y piensa que al usar la más 


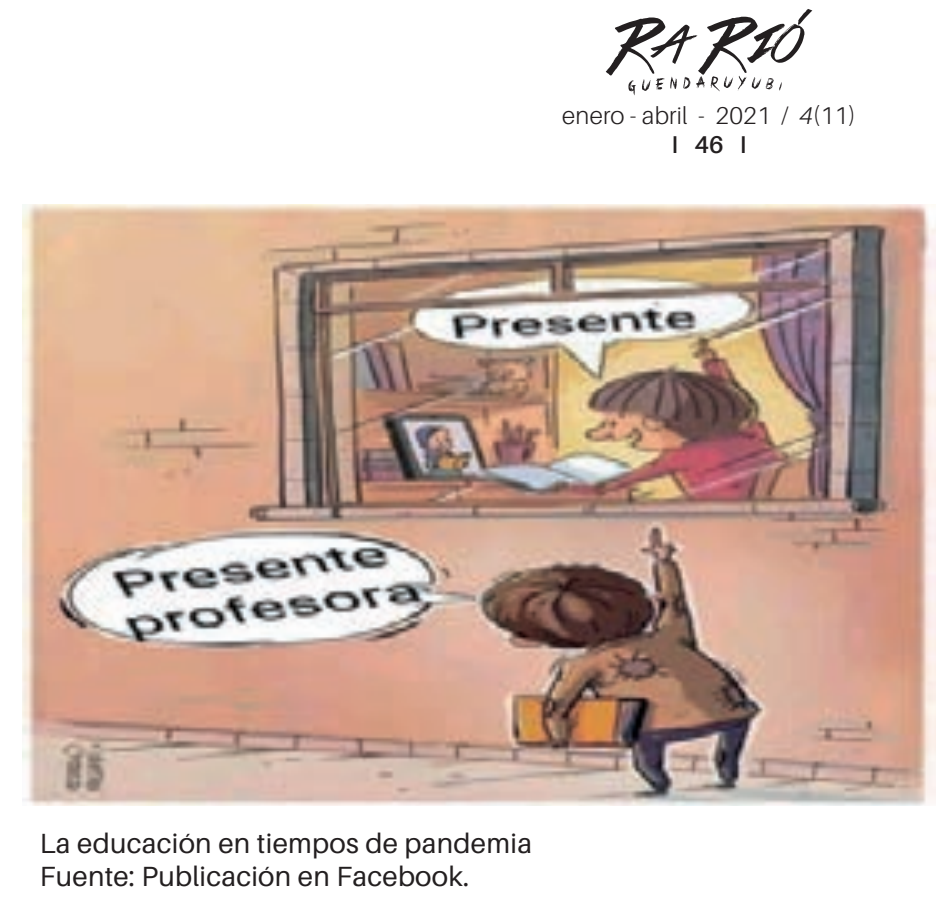

sofisticada tecnología se hará la diferencia para lograr los objetivos educativos, estará condenando su desempeño al fracaso. La tecnología es un medio, no un fin, se trata de un recurso valioso y atractivo, pero el contacto presencial no se reemplaza con nada, dado que la educación es una profesión típica de lo humano, sucede entre humanos, por humanos y para seres humanos.

Esta dinámica propia de la práctica docente de hacer el doble de esfuerzo por superar las limitaciones de la educación virtual, convierte a esta labor en una tarea hiperagotadora, porque los trabajos se incrementan; además, es frustrante porque no existe certeza de que los objetivos se estén logrando al lidiar en una batalla permanente contra el tiempo para desarrollar todos los temas y visualizar alguno que otro aprendizaje en el camino, aunado a las propias carencias de recursos a nivel personal y familiar. Otro reto más que queda en la agenda es la posibilidad de adaptar el currículo formal a estas nuevas formas de enseñanza y aprendizaje.

En este tenor, debemos pensar y asimilar la idea de que nuestro futuro como actores y actrices del proceso educativo es incierto y que las cifras reveladoras del calentamiento global y cambio climático en la época que nos tocó vivir nos instan a adquirir habilidades y recursos para afrontar situaciones como el COVID-19.

Es necesario darnos cuenta de que desde el ámbito social estamos presenciando el fin de una era, lo que determina para la educación una forma diferente de mirar la formación académica de niños, niñas y adolescentes, haciendo uso de recursos y herramientas que tengamos a nuestro alcance, siempre priorizando la función sustantiva de lo educativo. 

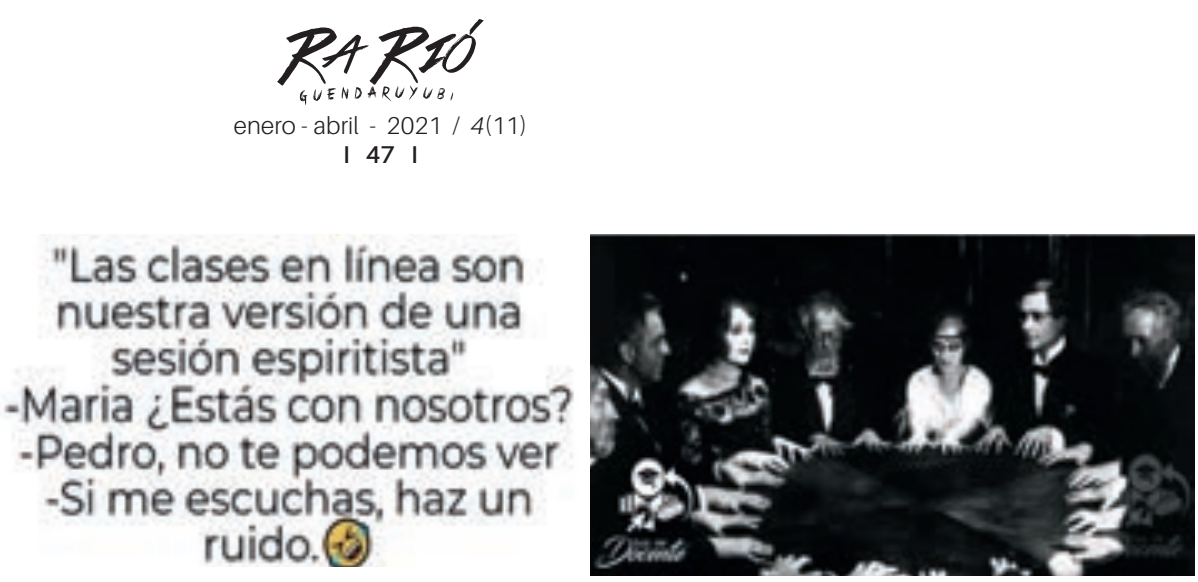

Meme sobre las clases en línea

Fuente: Guía del docente, 2020.

En palabras de Hernández, ante esta contingencia se visualiza que la educación del futuro o educación pospandemia se centrará en los medios digitales, pero también se reconoce que se necesita de la enseñanza presencial. "Quiero pensar que la educación del futuro no será en confinamiento. Sí se necesitan los medios tecnológicos. Esta situación nos acerca a la educación del futuro, pero no nos define" (Hernández, 2020). En este sentido, tal vez la educación del futuro se desarrolle a partir de un modelo híbrido, combinando la presencialidad con la virtualidad.

No queda más que esperar y en ese futuro, utilizar lo más valioso que nos queda, dar cuenta de las opiniones y reflexiones que podamos aportar desde la trinchera que nos toque para el mejoramiento de la formación de las nuevas generaciones; se lo debemos, siempre con el compromiso y entrega que esta noble tarea demanda.

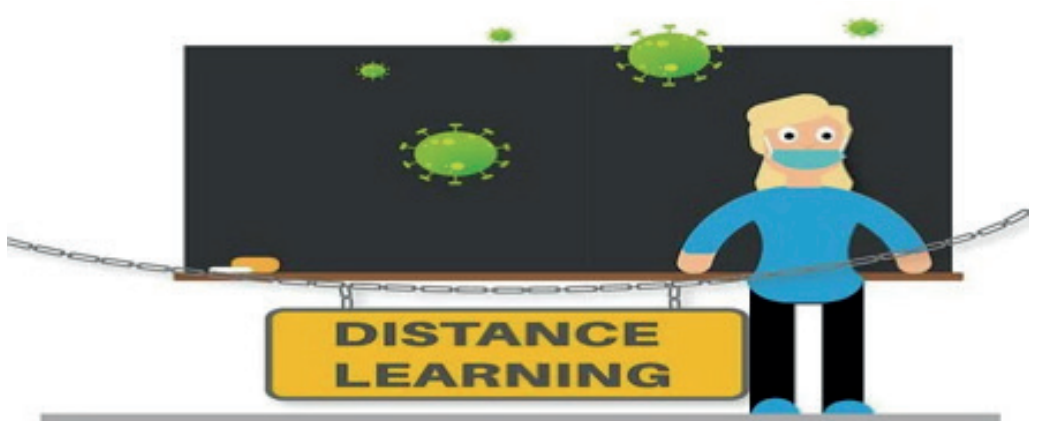

El aprendizaje a distancia

Fuente: Pixabay, 2020.

Referencias

Hernández, F.G.E (2020, 16 de junio). El campo educativo en el contexto actual. Videoconferencia realizada a través de la plataforma Zoom. 


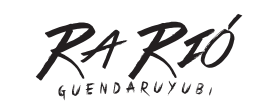

enero - abril - $2021 / 4(11)$

I 48 I

Impulso Creativo/Vulkan Visuals. (2020, 18 de mayo). La Tierra nos está hablando. Recuperado de https://www.youtube.com/watch?v=kWTHU8kXF-c

Instituto Nacional de Estadística y Geografía (INEGI). (2019). Encuesta Nacional sobre Disponibilidad y Uso de Tecnologías de la Información en los Hogares (ENDUTIH). Recuperado de https://www.inegi.org.mx/contenidos/saladeprensa/boletines/2020/ OtrTemEcon/ENDUTIH_2019.pdf

López Calva, Juan Martín (19 de noviembre de 2020). Videoconferencia Re-danzar el mundo. Panorama global del maestro en el 2020, realizada a través de la plataforma Zoom. Recuperado de https://www.youtube.com/watch?v=ITb5yWBWfhs

Puiggrós, A., Arroyo, J. P., Aguilar Villanueva, L. \& López, N. (2020, 28 de mayo). Primer Foro Virtual. Los Sistemas Educativos Nacionales ante situaciones de emergencia. Aprendizajes de la pandemia de la COVID-19 y perspectivas para el futuro. México: Comisión Nacional para la Mejora Continua de la Educación (MEJOREDU). Recuperado de https://www. youtube.com/watch?v=lyJbsMS5kZc

TED Ideas worth spreading (2007). Sugata Mitra muestra cómo los niños se enseñan a sí mismos. Recuperado de https://www.ted.com/talks/sugata_mitra_kids_can_teach_themselves/ transcript?language=es\#t-994063 